\title{
Proximal Tibial Fracture after Bone-Patellar Tendon-Bone Autograft Harvesting in Anterior Crutiate Ligament Reconstruction: Literature Review and Report of Two Cases
}

\author{
Akram M. Aldawoudy1,2, Galal G. Elfatarany¹, Ahmed M. Badr¹, Eiman Soliman³ \\ ${ }^{1}$ Alexknee Center, Alexandria, Egypt \\ ${ }^{2}$ Orthopedic Department, Alexandria University Student Hospital, Alexandria, Egypt \\ ${ }^{3}$ Internal Medicine Rheumatology and Clinical Immunology, Alexandria Faculty of Medicine, Alexandria, Egypt \\ Email: galalelfatarany@gmail.com
}

How to cite this paper: Aldawoudy, A.M., Elfatarany, G.G., Badr, A.M. and Soliman, E. (2018) Proximal Tibial Fracture after Bone-Patellar Tendon-Bone Autograft Harvesting in Anterior Crutiate Ligament Reconstruction: Literature Review and Report of Two Cases. Surgical Science, 9, 165-173. https://doi.org/10.4236/ss.2018.95019

Received: February 18, 2018

Accepted: May 7, 2018

Published: May 10, 2018

Copyright $\odot 2018$ by authors and Scientific Research Publishing Inc. This work is licensed under the Creative Commons Attribution International License (CC BY 4.0).

http://creativecommons.org/licenses/by/4.0/

\begin{abstract}
ACL reconstruction surgery shows low incidence of complications (1.38\%) like DVT, Infection, patellar tendon rupture and fractures whether in the proximal tibia or the distal femur. We hereby present two cases that have had the rare complication of proximal tibial fracture. The circumstances of each case are discussed together with discussion of similar cases described in the literature. We tried in the end to have an idea about the possible causes and how to prevent such a devastating event. Case presentation: Case 1 shows a 38-year-old female patient who had an ACL reconstruction surgery by BTB graft followed by iatrogenic proximal tibial fracture managed by open reduction internal fixation by $\mathrm{T}$ plate and screws. Case 2 shows a 28 -year-old male patient who had an ACL reconstruction surgery by BTB graft also followed by Proximal tibial fracture 3 weeks later managed by open reduction internal fixation using a locked plate and screws. Discussion: Fractures are a rare complication that may follow ACL reconstruction surgery. These fractures can be femoral or tibial that may be due to the reduction of the bone strength as a result of the drill holes which behave as a stress riser. We also suggest that usage of osteotomes during grafting plays a role in these fractures. Management of these fractures is either by open reduction and internal fixation or closed reduction. What is known about the subject: Patellar fractures are a known complication of ACL reconstruction using patellar tendon graft. What this study adds to the existing knowledge: Raising the Knowledge that also the tibia can be fractured as a complication of patellar tendon harvesting not only the patella.
\end{abstract}




\section{Keywords}

BTB, Proximal Tibial Fracture, ACL Reconstruction, Complications

\section{Introduction}

The surgical technique of anterior cruciate ligament (ACL) reconstruction has changed over the last few years as a result of improved understanding of ligament biomechanics, anatomy and device development [1] and also more knowledge about the complications of surgery that may occur intra or post operatively. These complications may be general, like DVT and Pneumonia [2] or specific to ACL reconstruction surgical procedure like anterior knee pains [3] and fractures around the knee [4] [5] [6] [7].

The choice of the graft depends on different patient's and surgeon's factors [8]. However, it was found that patellar tendon (PT) autografts produce a more statically stable knee [3] and less failure rate and risk of early revision than hamstrings tendons (HT) [9] [10].

Bone-Tendon-Bone (BTB) fixation to the tibia using press-fit technique in ACL reconstruction is an efficient procedure in comparison with the interference screw fixation. It has unlimited bone-to-bone healing, no need for removal of hardware, ease for revision and cost effectiveness [11] [12].

PT ACL reconstruction technique in general has some potential complications like anterior knee pains, patellar tendon ruptures[6] and fractures that may be patellar [13] tibial [4] [5] [6] [7] [14] and femoral [15] were reported.

In this report, we present 2 cases of proximal tibial fractures after bone patellar tendon bone graft harvesting and comment on the possible etiology and preventive measures.

\section{Case 1}

A 38-year-old overweight female patient presented by left knee pain after twisting injury and falling down during walking one month before presentation. On clinical examination there was mild effusion, increased antero-posterior laxity and medial laxity with end points and flexion attitude of about $5^{\circ}$. Her MRI revealed completely torn ACL (Figure 1).

The patient underwent arthroscopic ACL reconstruction using ipsilateral BTB graft and debridement of partial tear of lateral meniscus root (Figure 2).

While harvesting of the graft from the tibial attachment, iatrogenic fracture of the proximal tibial extremity occurred. The graft was harvested using osteotomes and it was $17 \mathrm{~mm}$ based from the tibial side. The graft was fixed tibially using press-fit graft tunnel mismatch technique and femorally by bio-interference screw of $9 \times 28 \mathrm{~mm}$ size (Figure 3 ).

Fluoroscopic-assisted fixation of the fracture was done using T-locked plate through extension of the harvesting incision. Intraoperative examination confirmed graft stability and postoperative radiographs showed correct placement of 


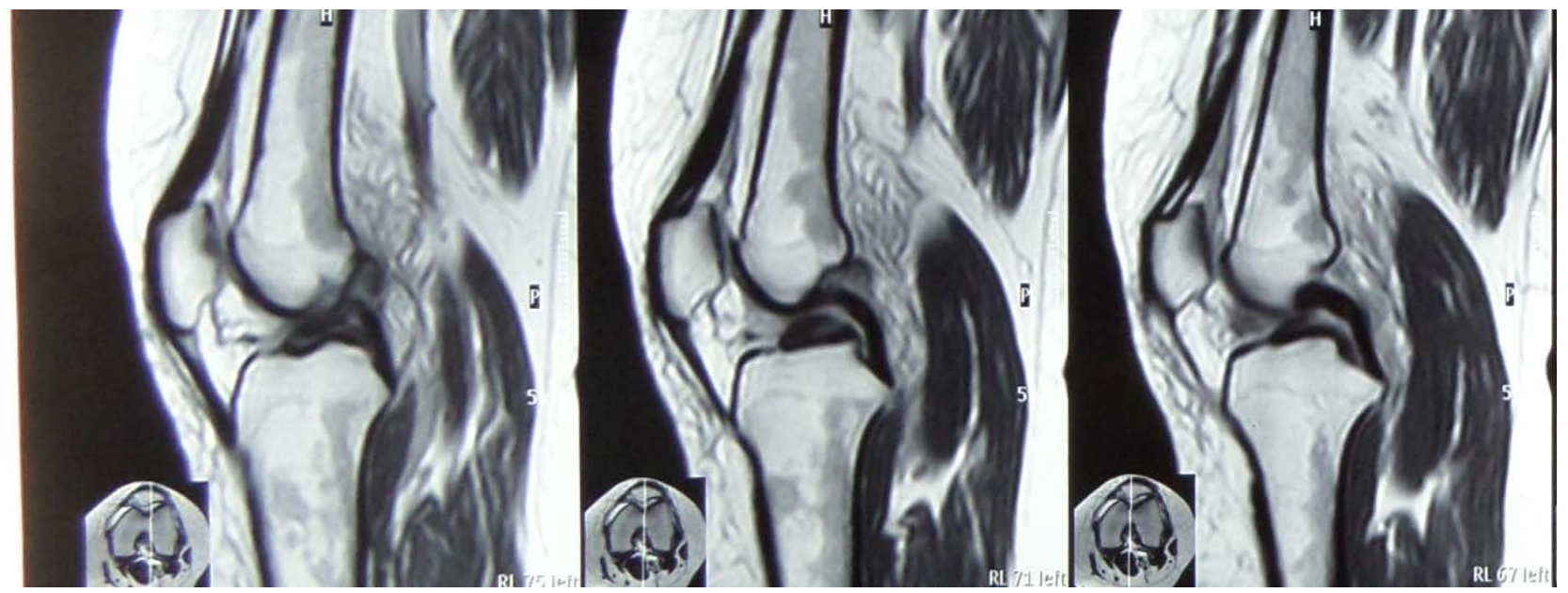

Figure 1. MRI (T1) left knee revealed torn ACL.

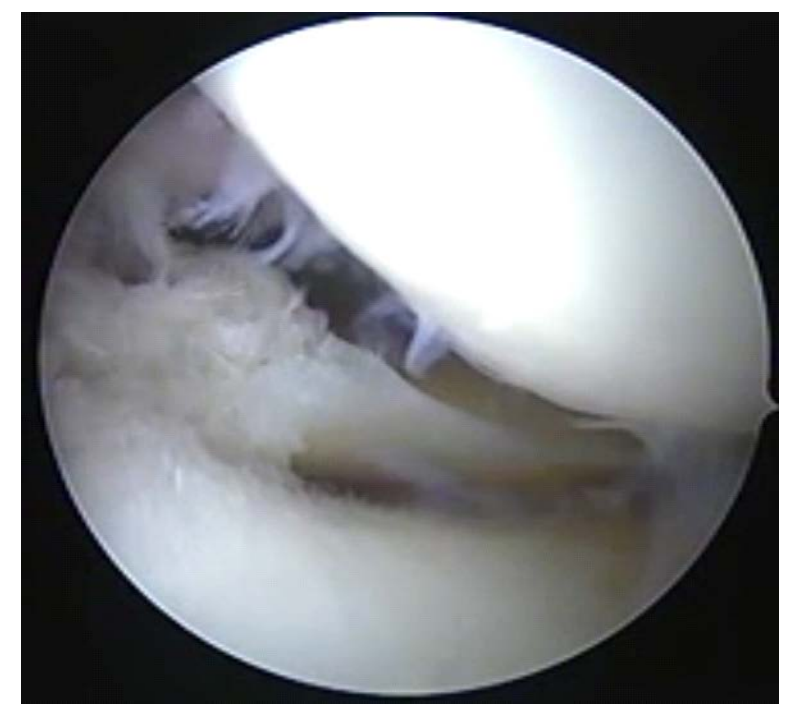

Figure 2. Artroscopic appearance of partial tear of the lateral meniscal root.

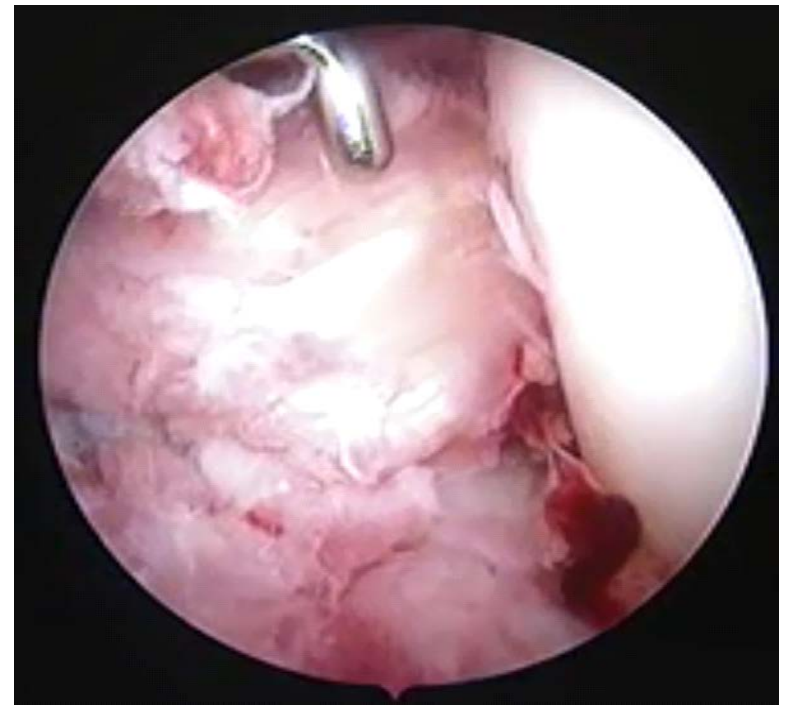

Figure 3. Arthroscopic appearance of the ACL BTB graft. 


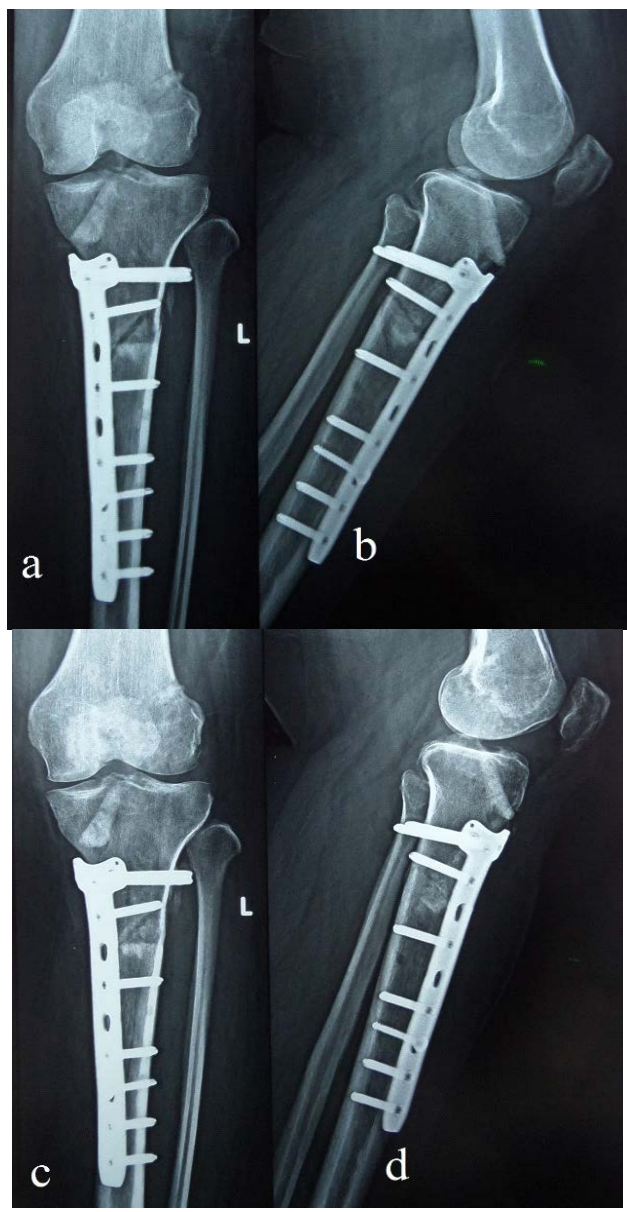

Figure 4. ((a), (b)) Plain X-ray of the left knee AP and lateral views one month post-operatively, showing good position of the graft, intact tibial tunnel and good reduction of the iatrogenic tibial fracture. ((c), (d)) AP and lateral views three months post-operative showing good healing of the fracture.

the graft and good reduction of the fracture (Figure 4(a) \& Figure 4(b)).

Three months post-operatively, the patient flexion range was up to $120^{\circ}$ with $5^{\circ}$ of extension lag and the X-ray showed fully united fracture (Figure 4 (c) \& Figure 4(d)). The patient was advised to continue her physiotherapy protocol and to start partial weight bearing. Full healing occurred over the following few weeks.

The last follow up of the patients was 3 months from the operation and she was doing great with no pain on the fracture site and full range of motion but the muscle power was still not full.

She stayed in the Hospital for 1 day which is longer that our daily routine ACL reconstructions cases which stay for $6-10$ hours in the hospital.

The total cost of the operation was 24,000 LE which is more than the normal cost of just ACL reconstruction cases.

\section{Case 2}

A 28-year-old male patient presented by left knee pain after stress valgus injury 
during playing football one week before presentation. On clinical examination there was mild effusion, tenderness medially, increased anteroposterior laxity and there was mild varus deformity bilaterally and generalized hyperlaxity. His MRI revealed torn ACL (Figure 5).

The patient underwent arthroscopic reconstruction of the ACL by BTB graft which was fixed femorally by interference screw $9 \times 28 \mathrm{~mm}$ and tibially using press-fit technique, posterior horn lateral meniscus tear was discovered and partial menisectomy was done.

Three weeks later, the patient presented again by sudden giving way and severe pain after mild twisting injury while getting down stairs, his X-ray revealed oblique extra-articular fracture of the left proximal tibia and fibula (Figure 6).

He underwent open reduction and internal fixation of the fracture by medial locked plate. One week later, the wound became infected and he underwent surgical debridement and a culture was taken. His culture revealed no growth and the wound closed after two weeks.

Six weeks after the fixation, the patient had full range of motion, his X-ray

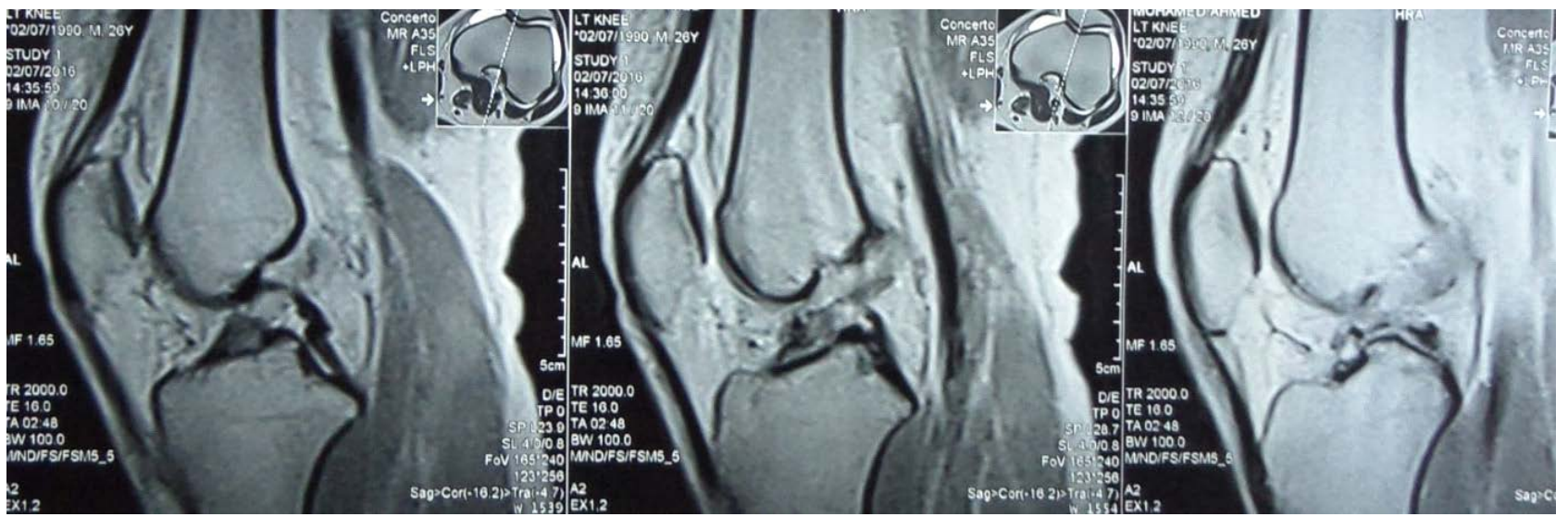

Figure 5. MRI left knee revealed torn ACL.

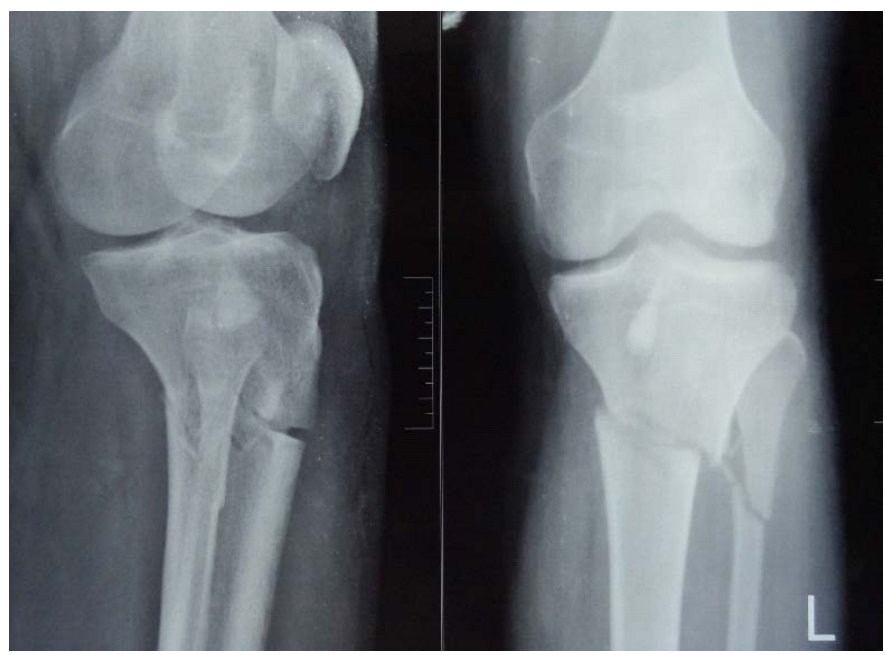

Figure 6. AP and lateral views showing fracture of the proximal tibia three weeks after ACL reconstruction by BTB graft with good position of the graft. 


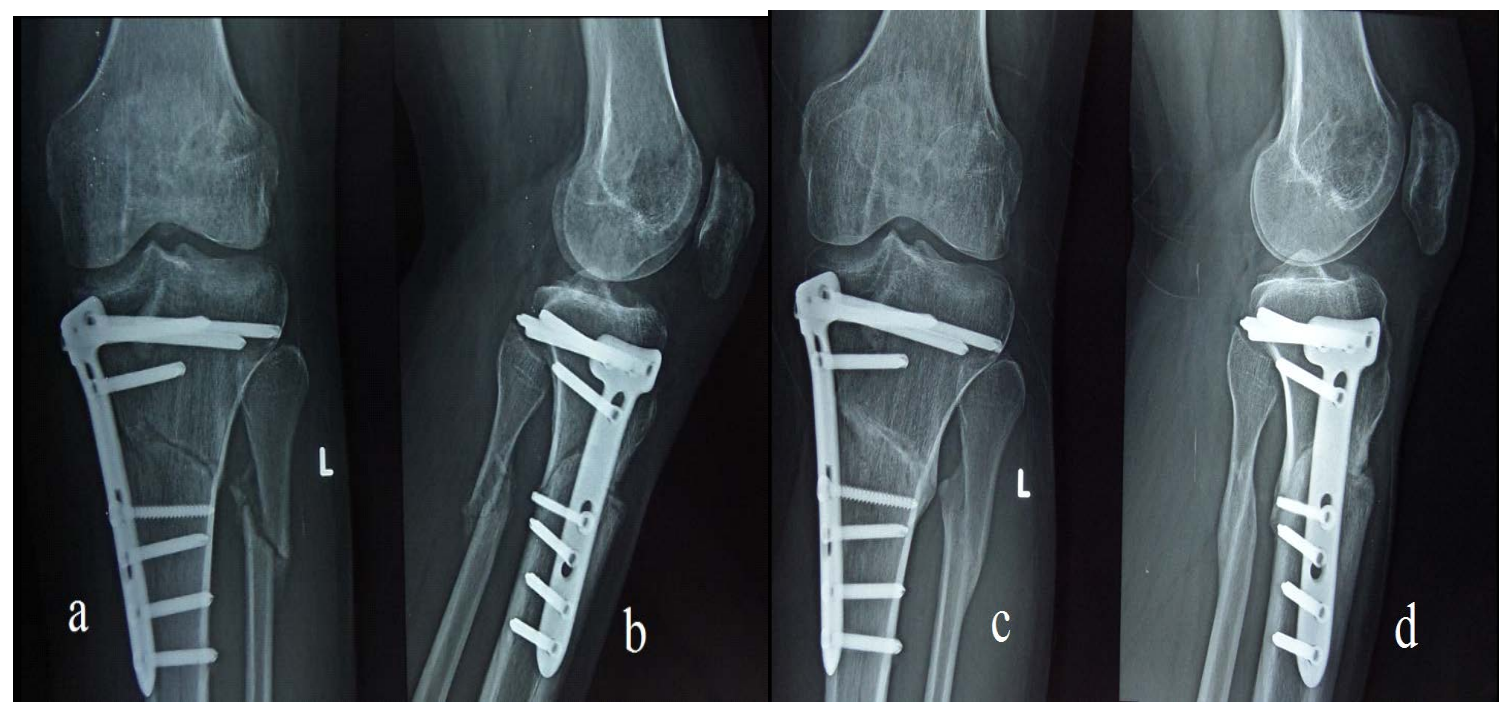

Figure 7. ((a), (b)) Plain X-rays of the left knee, AP and lateral views six weeks post-operatively, ((c), (d)) AP and lateral views five months postoperatively showing complete union of the fracture.

showed starting union (Figure 7(a) \& Figure 7(b)) and he started partial weight bearing. After five months, he was fully weight bearing with stable knee and his PXR showed complete healing.

The last follow up of this patient was 1 year post operative and he showed no tenderness on the fracture site, full range of motion and full muscle power.

He stayed after the second operation for 1 day which is normal in the open reduction internal fixation of the proximal operations.

The total cost of the 2 operations were $31,000 \mathrm{LE}$

\begin{tabular}{ccc}
\hline & Case 1 & Case 2 \\
\hline Sex & Female & Male \\
Age & 38 & 29 \\
Occupation & House wife & Engineer \\
Side & Left & Left \\
$1^{\text {st diagnosis }}$ & Torn ACL & Torn ACL \\
Fracture time & Intraoperative & 3 weeks Postoperative \\
Management & Open reduction and Internal Fixation by & Open reduction and Internal Fixation by \\
Plate and screws & Plate and screws \\
Complications & None & Superficial Infection \\
Last X-ray & 3 months post-Operative X-ray shows & 3 Months post-operative X-ray shows \\
shows & Complete healing & complete healing \\
Total Cost & 24000 LE & 31000 LE \\
\hline
\end{tabular}

\section{Discussion}

Tibial fractures after ACL reconstruction are rare and have been scarcely reported since 2001 [4] [6]. 
They can affect one of the 2 tibial Plateaus [4] [6] [16] both condyles [14] and the proximal tibial area [5] [7] [17].

These fractures can occur with the different types of grafts. It can occur with PT graft [5] [6] [7], Achilles tendon allograft [16], or even HT grafts [17] but it is more common with PT graft.

Delcogliano [6] reported Lateral Tibial Plateau fracture with minimal trauma 7 months after ACL reconstruction in a 27-year-old male patient using PT graft fixed tribally and femorally by interference screws. He proposed that transosseous drilling and screw placement can act as sites of decreased bone strength even several months after surgery

Voos [7] reported oblique metaphyseal tibial fracture with extension from the tibial donor site into the articular surface 9 weeks after ACL reconstruction of a 43 years old female patient using PT graft. This graft was fixed on the tibial and femoral sides by interference screws. He suggested that PT autografts may weaken the proximal tibia in addition to the weakness already occurring with bone tunneling. He also suggested that the age of the patient could have played a role, as he may have been suffering from osteoporosis.

Carneiro [5] also reported extra-articular fracture of the proximal tibia 4 months after ACL reconstruction of a 17-year-old male patient using PT graft. This graft was also fixed on the tibial and femoral sides by interference screws. He suggested that harvesting the tibial bone plug could have increased the susceptibility of the proximal tibia to fracturing. His theory behind that was that the bone that was drilled through to create the tibial tunnel, was subject to stress and may have lead to fatigue when torsional forces were applied to it during flexion.

Sundaram [17] stated that the tibial tunnel alone may behave as a stress riser which can significantly reduce bone strength. Bae [4] also suggested the same reason for reducing strength at the level of proximal tibial metaphysis.

It has been well documented in the orthopedic literature that bone defects, such as screw holes after drilling and implant removal, can collect stresses and significantly decrease bone resistance to bending and torsional forces [18]

The current 2 case report were managed by Open reduction and internal fixation with locked plates and screws. Open reduction and internal fixation using plate and screws are described in most of the cases reported in the literature [5] [6] [7]. Mithöfer [14] described minimal-invasive fracture fixation of an intra-articular tibial plateau fracture after anterior cruciate ligament reconstruction. Sundaram [17] Described plaster non-weight bearing cyndrical cast for 6 weeks, then functional weight bearing for another 6 weeks.

Consequently, we suggest that the cause of the fracture that happened on table with us in the 1st case may be the usage of osteotomes instead of the electric saw during harvesting of the PT graft especially on the tibial side, besides going too much distally as the patellar tendon was relatively short in this particular patient. In addition, the wide based tibial part of the graft $(17 \mathrm{~mm})$ and the drilling of the tibial tunnel itself constituted other reasonable factors towards easy fracturing. 
Regarding the 2nd case there is no clear reason for us why the fracture happened 3 weeks postoperatively apart from having both wide based graft besides the tibial tunnel working as stress-riser.

\section{References}

[1] Tibor, L., Chan, P.H., Funahashi, T.T., Wyatt, R., Maletis, G.B. and Inacio, M.C.S. (2016) Surgical Technique Trends in Primary ACL Reconstruction from 2007 to 2014. The Journal of Bone and Joint Surgery, 98, 1079-1089. https://doi.org/10.2106/JBJS.15.00881

[2] Cvetanovich, G.L., Chalmers, P.N., Verma, N.N., Cole, B.J. and Bach Jr., B.R. (2016) Risk Factors for Short-Term Complications of Anterior Cruciate Ligament Reconstruction in the United States. The American Journal of Sports Medicine, 44, 618-624. https://doi.org/10.1177/0363546515622414

[3] Mohtadi, N.G., Chan, D.S., Dainty, K.N. and Whelan, D.B. (2011) Patellar Tendon versus Hamstring Tendon Autograft for Anterior Cruciate Ligament Rupture in Adults. Cochrane Database of Systematic Reviews, 9, CD005960. https://doi.org/10.1002/14651858.CD005960.pub2

[4] Bae, K.C., Cho, C.H., Lee, K.J. and Jeon, J.H. (2015) Bilateral Medial Tibial Plateau Fracture after Arthroscopic Anterior Cruciate Ligament Reconstruction. Knee Surgery \& Related Research, 27, 129-132. https://doi.org/10.5792/ksrr.2015.27.2.129

[5] Carniero, M.O., Montiero, T.A., Bueno, M.R.Z. and Augustin Jr., J.L. (2015) Fracture of the Proximal Extremity of the Tibia after Anterior Cruciate Ligament Reconstruction: Case Report. Revista Brasileira de Ortopedia, 50, 356-359. https://doi.org/10.1016/j.rboe.2015.04.009

[6] Delcogliano, A., Chiossi, S., Caporaso, A., Franzese, S. and Menghi, A. (2001) Tibial Plateau Fracture after Arthroscopic Anterior Cruciate Ligament Reconstruction. The Journal of Arthroscopic and Related Surgery, 17, 1-5. https://doi.org/10.1053/jars.2001.19450

[7] Voos, J.E., Drakos, M.C., Dean, G., Lorich, D.G. and Fealy, S. (2008) Proximal Tibia Fracture After Anterior Cruciate Ligament Reconstruction Using Bone-Patellar Tendon-Bone Autograft: A Case Report. Hospital for Special Surgery Journal, 4, 20-24.

[8] Joyce, C.d., Randall, K.L., Mariscalco, M.W., Magnussen, R.A. and Flanigan, D.C. (2016) Bone-Patellar Tendon-Bone Versus Soft-Tissue Allograft for Anterior Cruciate Ligament Reconstruction: A Systematic Review. The Journal of Arthroscopic and Related Surgery, 32, 394-402. https://doi.org/10.1016/j.arthro.2015.08.003

[9] Rahr-Wagner, L., Thillemann, T.M., Pedersen, A.B. and Lind, M. (2014) Comparison of Hamstring Tendon and Patellar Tendon Grafts in Anterior Cruciate Ligament Reconstruction in a Nationwide Population-Based Cohort Study. The American Journal of Sports Medicine, 42, 278-284. https://doi.org/10.1177/0363546513509220

[10] Reinhardt, K.R., Hetsroni, I. and Marx, R.G. (2010) Graft Selection for Anterior Cruciate Ligament Reconstruction: A Level I Systematic Review Comparing Failure Rates and Functional Outcomes. Orthopedic Clinics of North America, 42, 249-262. https://doi.org/10.1016/j.ocl.2009.12.009

[11] Sarzaeem, M.M., Najafi, F., Razi, M. and Najafi, M.A. (2014) ACL Reconstruction Using Bone-Patella Tendon-Bone Autograft: Press-Fit Technique vs. Interference Screw Fixation. Archives of Orthopaedic and Trauma Surgery, 134, 955-962. https://doi.org/10.1007/s00402-014-1999-3 
[12] Smith, P.A., Stannard, J.P., Pfeiffer, F.M., Kuroki, K., Bozynski, C.C. and Cook, J.L. (2016) Suspensory Versus Interference Screw Fixation for Arthroscopic Anterior Cruciate Ligament Reconstruction in a Translational Large-Animal Model. The Journal of Arthroscopic and Related Surgery, 32, 1086-1097. https://doi.org/10.1016/j.arthro.2015.11.026

[13] Christen, B. and Jakob, R.P. (1992) Fractures Associated with Patellar Ligament Grafts in Crutiate Ligament Surgery. The Journal of Bone and Joint Surgery. British Volume, 74-B, 617-619. https://doi.org/10.1302/0301-620X.74B4.1624526

[14] Mithöfer, K., Gill, T.J. and Vrahas, M.S. (2004) Tibial Plateau Fracture Following Anterior Cruciate Ligament Reconstruction. Knee Surgery, Sports Traumatology, Arthroscopy, 12, pp. 325-328. https://doi.org/10.1007/s00167-003-0445-9

[15] Wiener, D.F. and Siliski, J.M. (1996) Distal Femoral Shaft Fracture: A Complication of Endoscopic Anterior Cruciate Ligament Reconstruction. The American Journal of Sports Medicine, 24, 244-247. https://doi.org/10.1177/036354659602400224

[16] El-Hage, Z.M., Mohammed, A., Griffiths, D. and Richardson, J.B. (1998) Tibial Plateau Fracture Following Allograft Anterior Cruciate Ligament (ACL) Reconstruction. International Journal of the Care of the Injured, 29, 72-74. https://doi.org/10.1016/S0020-1383(97)00130-7

[17] Sundaram, R.O., Cohen, D. and Barton-Hanson, N. (2006) Tibial Plateau Fracture Following Gracilis-Semitendinosus Anterior Cruciate Ligament Reconstruction: The Tibial Tunnel Stress-Riser. The Knee, 13, 238-240. https://doi.org/10.1016/j.knee.2005.08.009

[18] Johnson, B.A. and Fallat, L.M. (1997) The Effect of Screw Holes on Bone Strength. Journal of Foot and Ankle Surgery, 36, 446-451. https://doi.org/10.1016/S1067-2516(97)80097-X 